\title{
The inspiratory "squawk" in extrinsic allergic alveolitis and other pulmonary fibroses
}

\author{
JE EARIS, K MARSH, MG PEARSON, CM OGILVIE \\ From the Cardiothoracic Centre, Broadgreen Hospital, Liverpool
}

ABSTRACT An inspiratory musical sound ("squawk") was recorded in 14 patients with diffuse pulmonary fibrosis. These were divided into two groups: nine patients suffering from extrinsic allergic alveolitis (seven with bird fancier's lung and two with farmer's lung) and five patients with pulmonary fibrosis due to other causes, including rheumatoid disease, Wegener's granulomatosis, systemic sclerosis, and sarcoidosis. Clinical studies and phonopneumographic analysis of 10 consecutive squawks in each patient showed that the sound in the group with extrinsic allergic alveolitis was of shorter duration, occurred later in inspiration, and tended to be of higher frequency than the sound heard in the other group. Inspiratory crackles were present in all patients and in eight a single loud crackle preceded the squawk. We suggest that squawks, like crackles, result from the opening of airways and that the differences between the squawks in the two groups may reflect the size of the affected airways.

Laennec described a late inspiratory wheeze as "le cri d'un petit oiseau" and Forgacs noted this sign in some patients with cryptogenic fibrosing alveolitis. ${ }^{1}$ Geddes and others described a similar sound in six patients with rheumatoid lung disease. ${ }^{2}$ For some time we have been aware of a short, isolated inspiratory sound ("squawk") occurring in patients with pulmonary fibrosis due to various causes. During the past 12 months we have found this physical sign in 14 such patients, of whom nine were suffering from extrinsic allergic alveolitis. In this paper we describe the clinical and phonopneumographic features of the inspiratory squawk in these 14 patients.

\section{Methods}

The 14 patients consisted of seven men and seven women with a mean age of 49 years (range 25-73 years). They were all suffering from conditions associated with diffuse pulmonary fibrosis. In nine cases this was due to extrinsic allergic alveolitis, seven of the patients having bird fancier's lung (three kept budgerigars, three pigeons, and one a parrot) and two having farmer's lung. Of the other pulmonary fibroses, one was associated with rheumatoid disease, one with Wegener's granulomatosis, one with systemic sclerosis, and one with sarcoidosis and one was idiopathic. The diagnosis of extrinsic allergic alveolitis was based on a

Address for reprint requests: Dr JE Earis, Fazakerley Hospital, Longmoor Lane, Liverpool 9. history of appropriate exposure and at least four of the following findings: late inspiratory crackles, radiographic shadows predominantly over the upper zones, a restrictive spirometric pattern, a reduced gas transfer factor, and the presence of precipitins (avian or farmer's lung) in the blood.

Patients were examined on at least two separate occasions. The site, duration, pitch, and position in inspiration of the squawks were recorded, together with the effects of different breathing patterns, exercise, and posture. Chest radiography and measurement of ventilatory capacity, lung volumes, and transfer factor were performed at each visit, with other tests as clinically indicated.

The sound recordings were made with the patient sitting. A crystal suction microphone was placed over the area of the squawk (usually the anterior chest wall in the upper zone). The output from the microphone was amplified (Cambridge phono amplifier) without filtration. The signal was monitored with headphones and recorded on one channel of a four-channel frequency-modulated tape recorder (Hewlett Packard). Simultaneously, air flow at the mouth was measured with an Ohio dry spirometer, the signal from which was recorded on the second channel of the tape recorder. The sound and flow signals were then played back through an ink jet recorder to give a visual tracing (fig 1). Frequency was measured from the tracing by counting the number of oscillations in a given time (paper speed $500 \mathrm{~mm} \mathrm{~s}$ ). 


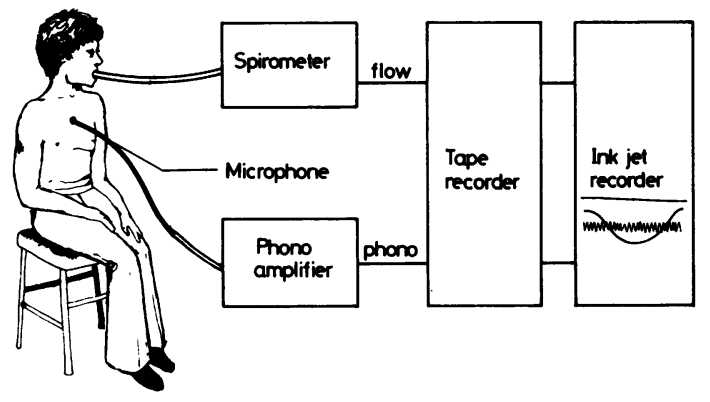

Fig 1 Apparatus used for simultaneous recording of breath sounds and inspiratory flow rates.

\section{Results}

\section{CLINICAL FINDINGS}

In 13 of the 14 patients a high-pitched inspiratory sound ("squawk") was heard over the upper chest, while in the remaining patient (who had rheumatoid disease) it was maximal over the lower chest. In four of the patients with extrinsic allergic alveolitis and three of the others it was also audible at the mouth. The sound was most easily heard with the patient semirecumbent and breathing deeply. Very deep breathing, coughing, and exercise, however, often abolished the sound, in some cases for several minutes. The squawk was intermittent, particularly in the patients with extrinsic allergic alveolitis, when it sometimes occurred only once or twice a minute. Despite this breath-to-breath variation the sign was remarkably constant from one clinic visit to the next. In one patient (with systemic sclerosis) it had been noted at every visit for 10 years, in three patients (with sarcoidosis, Wegener's granulomatosis, and extrinsic allergic alveolitis) for four years, and in one (with pigeon fancier's lung) for one year. Although in all patients the inspiratory squawk was of short duration it was noticeably briefer in the group with extrinsic allergic alveolitis. Its position during inspiration also differed between the groups: in those with extrinsic allergic alveolitis it occurred late while in the other patients it was in the early or middle part of inspiration. Inspiratory crackles preceded the sign in all the patients.

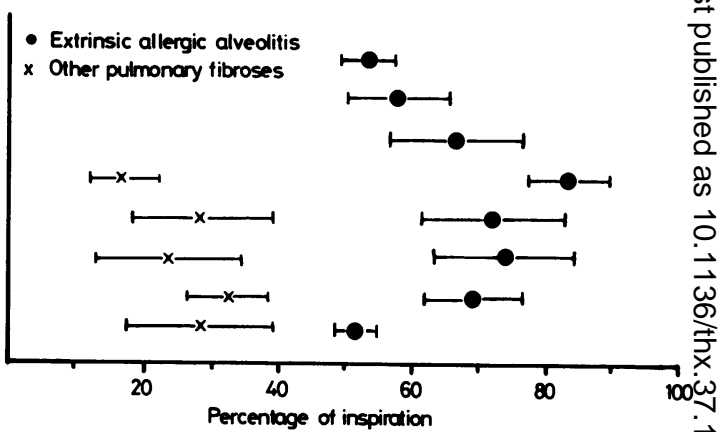

Fig 2 Onset of the "squawks" in inspiration: each circle or $\vec{N}$ cross represents the mean of 10 consecutive squawks in an individual patient and the bars represent $\pm 1 S D$.

\section{PHONOPNEUMOGRAPHIC ANALYSIS}

Ten consecutive squawks were analysed from eac $P$ individual patient according to their frequency $\frac{\mathbb{2}}{3}$ duration, and position in inspiration (with the excepo tion of one patient with farmer's lung on whom data were insufficient). The mean values for each of theseo variables in the two groups of patients are seen in the table. In the extrinsic allergic alveolitis group the squawks were of higher frequency (difference not statistically significant) and shorter duration $(p<0.01)$ and occurred later in inspiration $(\mathrm{p}<0.01)$ than those in the group with other forms of pulmonary fibrosis (statistical analysis was by Wilcoxon's rank sum test). Figure 2 shows the time of onset of the sound in the individual patients. The sound began in the second half of inspiration in the extrinsic allergic alveolitis group and in the first half of inspiration in the other group.

In the two groups taken together, there was a significant relationship (on the basis of linear regres ${ }_{\sigma}^{\times}$ sion analysis) between the duration and the fre quency of the squawk-sounds of higher frequencis being of shorter duration $(r=0.71, p<0.01)$.

A typical phonopneumogram of the short, high-o pitched, late inspiratory squawk in a patient with extrinsic allergic alveolitis is seen in figure 3 while? the longer, earlier inspiratory squawk in the patien with sarcoidosis is seen in figure 4 . The large crackles preceding these squawks was also noted in six other patients.

\begin{tabular}{|c|c|c|}
\hline & $\begin{array}{l}\text { Extrinsic allergic alveolitis } \\
(n=9)\end{array}$ & $\begin{array}{l}\text { Other pulmonary fibroses } \\
(n=5)\end{array}$ \\
\hline $\begin{array}{l}\text { Frequency (Hz) } \\
\text { Duration (s) } \\
\text { Position (\% of inspiration) }\end{array}$ & $\begin{aligned} 321 & \pm 76 \\
0 \cdot 09 & \pm 0.05 \\
65 & \pm 11\end{aligned}$ & 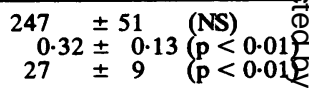 \\
\hline
\end{tabular}




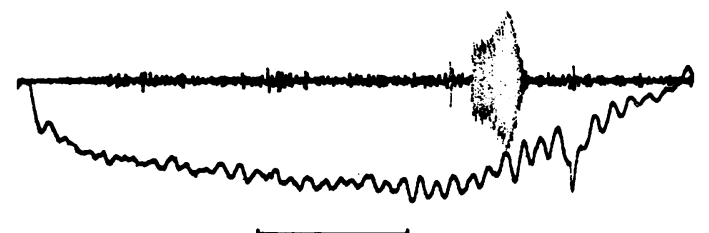

0.25 seconds

Fig 3 Phonopneumogram and simultaneous inspiratory flow trace of a patient with extrinsic allergic alveolitis due to exposure to a budgerigar; a crackle immediately precedes the "squawk."

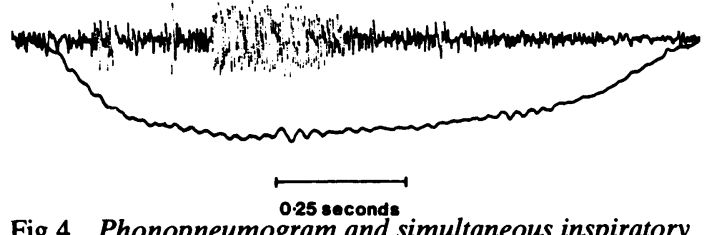

Fig 4 Phonopneumogram and simultaneous inspiratory flow trace of the patient with sarcoidosis; as in figure $3 a$ crackle immediately precedes the "squawk."

\section{Discussion}

Wheezing associated with airflow obstruction is commonly heard in both expiration and inspiration; isolated inspiratory wheezes are rare. Geddes and his coworkers described an isolated, high-pitched wheeze ("squeak") occurring in early-to-mid inspiration in six patients with severe airflow obstruction due to bronchiolitis obliterans associated with rheumatoid disease. ${ }^{2}$ The sound was sufficiently unusual to be considered as a useful sign suggesting the diagnosis of this rare condition.

In his monograph on lung sounds ${ }^{3}$ Forgacs also described inspiratory musical sounds in patients with various forms of diffuse interstitial pulmonary fibrosis (conditions not usually associated with airflow obstruction). He described a characteristic sequence of inspiratory monophonic wheezes of different pitches heard over the basal territories of the lung and also mentioned the occasional presence in cryptogenic fibrosing alveolitis of a single endinspiratory wheeze which sometimes followed a series of crackles. Forgacs postulated that these sounds were produced by airways in deflated areas of the lung opening late in inspiration; he considered that as a rule transition from the closed to the fully open state is instantaneous but if the walls remain momentarily in light contact the inrush of air through these barely open airways sets them into oscillation and produces a short wheeze.

In this study an inspiratory sound (the squawk) has been described in 14 patients with pulmonary fibrosis from a variety of causes. All patients had a restrictive rather than obstructive abnormality of ventilatory function. The sound was different from the sequential inspiratory wheezes described by
Forgacs, but apparently similar to the endinspiratory wheeze he heard in some patients with cryptogenic fibrosing alveolitis (though careful auscultation of the 20 patients with cryptogenic fibrosing alveolitis attending this unit during the past six months has failed to reveal this sign).

Squawks were associated with inspiratory crackles in all patients and in eight a loud crackle immediately preceded the squawk (figs 3 and 4 ). Thus the squawk seems likely to be generated by a mechanism similar to that postulated in sequential inspiratory wheezes-namely, air rushing into a small airway as it opens may occasionally set its walls and surrounding tissue into oscillation. If this explanation is indeed correct then it also indicates why the squawks occur at different times in inspiration in the two groups of patients. The late inspiratory crackles associated with fibrosing alveolitis are thought to arise from smaller, more peripheral airways that open late in inspiration while the earlier, coarser crackles in conditions such as bronchiectasis probably arise from more proximal airways opening at lower transpulmonary pressures. ${ }^{4}$ Thus squawks probably occur later in inspiration in patients with extrinsic allergic alveolitis because small peripheral airways are affected and earlier in inspiration in conditions which may affect larger airways (for example, bronchiectasis, sarcoidosis, and Wegener's granulomatosis).

The observation that the squawks heard in extrinsic allergic alveolitis are of shorter duration and tend to have a higher frequency than those occurring in the other patients (table 2), and the overall relationship between frequency and duration, may also be explained by the size of the affected airways. High-frequency sounds are known to require a smaller vibrating mass of tissue than sounds of lower pitch. ${ }^{3}$ Thus squawks occurring in more peripheral airways which have a small mass would tend to be of higher frequency and decay more rapidly than those occurring in larger airways. Moreover, small airways which open late in inspiration are probably subject to greater elastic forces and therefore higher tension than larger airways, and this might increase their tendency to vibrate more rapidly.

We suggest that the short, high-pitched late inspiratory squawk heard in patients suffering from extrinsic allergic alveolitis may be of value in suggesting this diagnosis, especially when-as in three of our nine cases-the chest radiograph is normal.

We are grateful to Dr A Harley, consultant cardiologist, and Mr K McMann, medical physics technician, for their advice and help in assembling the phonopneumography equipment. 


\section{References}

adults and its association with rheumatoid disease. $Q$

${ }^{1}$ Forgacs P. Crackles and wheezes. Lancet 1967;ii:203-5. Med 1977;184:427-43.

${ }^{2}$ Geddes DM, Corrin B, Brewerton DA, Davies RJ; Turner-Warwick M. Progressive airway obliteration in

${ }^{3}$ Forgacs P. Lung sounds. London: Baillière Tindall, 1978

${ }^{4}$ Nath AR, Capel LH: Lung crackles in bronchiectasis $\overrightarrow{\widetilde{D}}^{3}$ Thorax 1980;35:694-9. 